\title{
Research on E-sports session identification
}

\author{
Cheng $\mathrm{Yu}^{1}$, WanNing Zhu ${ }^{1,}$, LiuLei $\mathrm{Li}^{1}$, YuMeng Sun ${ }^{2}$, and Liqing Nong ${ }^{3}$ \\ ${ }^{1}$ Department of Software Engineering, Jinling Institute of Technology, 211100, Nanjing, China \\ 2 Department of Business English, Jiangsu University of Technology, 213001, Changzhou, China \\ ${ }^{3}$ Secretary major, Guangxi University of Finance and Economics, 530003, Nanning, China
}

\begin{abstract}
With the development of the e-sports, data analysis has gained more and more attention in the use of it. For the important problem that the analysis of behaviour patterns of the e-sports' individual character is not solved directly and solved by anyone, this paper has put forward the session partitioning algorithm based on heat maps of jungle which adopts the clustering prototype thinking that partitions spatial range in advance and then determines by the range of time threshold. Experiments show that it solves the problem of roles of the session which is difficult to be partitioned.
\end{abstract}

\section{Introduction}

Data analysis has gained more and more attention with the function in e-sports industry. [1] The professionalization of video games requires tools that are adapted to the development of the players' practice. In order to increase their performances, cyber-athletes should have these tools available, so that they can define aims, analyze strength and weakness, then draw up a plan. We show here that analysing tracks of video games leads to lines of strategic analysis: wisely using the knowledge stemming from tracks provides solutions for evaluating and steering the sporting practice. [1]Put forward a method aims at analysis of tracks of video games, and explains the influence which track charts of team position have on victory or failure.[2] Come up with association rules, that is the combination algorithm which is digged into the association rules effectively to get the character with higher winning percentage in the game.

Existing work of data analysis of the e-sports has an over magnified view. To competitions, the importance of teamwork is holding the balance, but the individual behaviour tend to decide the process of the game and thus affect its result. The analysis of Individual behavior patterns are of great importance to analyze preparations for game strategies, make personal coup and predict opponents' strategies. This problem is very essential but it has not been solved by anyone so far. The problem is also complex, and it can not be solved directly because the analysis of behavior [3] patterns of the character (the character in the paper is jungle) should go through following phases: acquisition of the time contained, sample set of position coordinates [4], data cleaning [5], identification of characters [6], session partitioning, analysis of behavior patterns and so on. This paper has studied session partitioning algorithm. Session is the original concept existing when users

* Corresponding author: zhuwanning@jit.edu.cn 
access web applications[7], but in this paper, it indicates some specific activities of some characters ongoing in the game such as jungle, counter jungle, GANK and so on. It is obvious that to the analysis of behaviour patterns of the character are necessarily based on the session rather than the coordinate vector set. Therefore how to conduct session partitioning according to the original sample set is becoming an urgently need to be solved issue. This paper has put forward the session partitioning algorithm based on heat maps of jungle which adopts the clustering prototype thinking that partitions spatial range in advance and then determines by the range of time threshold. It solves the problem of roles of the session which is difficult to be partitioned. On the basis of the test, it is able to partition session with $90 \%$ accuracy and this ultimately solve the problem of session partitioning of the character in e-sports.

\section{The session partitioning algorithm of E-sports}

\subsection{Prepared knowledge for the algorithm}

This paper has analysed the game of LOL[8] in which at the beginning the game, roles of both sides departure from the base and some soldiers exist at regular intervals. Soldiers go along fixed roads. There are three roads altogether, and except the base and the three main roads, there is an area called the jungle. During the game, we have at least one player of both sides act in the jungle (the behaviour is called jungling). Because of lack of vision, the behaviour of jungling tends to attack opposite side by surprise. (GANK)There are monsters in the jungle. The prime act of jungling at the beginning of the game is killing the monster. This prevent opposite side counter jungling and GANK. The analysis of behaviour patterns of jungling is extremely important due to the successful counter jungling and GANK can obtain great advantages for the team at the beginning of the game. [9]

Jungling is a main behavior in the jungle, but in consideration of some special behaviours that will happen at some time, we give the following definite. Over a period of time, enemies will invade the jungle to hamper progress of their rivals or seek an opportunity to kill them. This is the behaviour we called counter jungling. To reduce the consumption of killing monsters, the player of jungling sometimes select to bring the monster out of its area and then kill it. This is the behaviour we called pull, but sometimes players play a disorder (it means when pull the monster to an extreme distant, the monster back to its area and regenerates HP.)This is the behaviour we called the failure of pulling. When the hero of one side kills the hero of the other side, the scoreboard will give the killer one score for killing and give the killed one score for being killed. When the hero killed by monsters or soldiers, the scoreboard will give the hero one score for being killed and no hero will get the score. If one hero assist other heroes of the same team kill enemies, he will be given one score for one assist at the same time when the killer and the killed get the score. If the player of jungling stay in the jungle beside the main road for a long time, he must be seeking the opportunity to GANK. This behaviour we called squat for people. Heroes can use the function of backing to the base to return to top form and purchase or sell equipment because they sometimes in a bad state or need to update equipment.

\subsection{Algorithm introduction}

This paper put forward nine kinds of the session of jungling altogether. They are jungle, counter jungle, the failure of pulling, the death caused by monsters, generation of killing, generation of assists, GANK, squat and back to the base. The evidence of the classification is: jungling at one session must be moving at a certain range, once beyond, it must start 
another session. For example, the jungle and GANK are not happen in the range of the same map. Thus we can use the region of plane coordinate to directly distinguish the session of the large span of the map. However, the session that happens in the same range will not be distinguished by direct coordinate because the data heat are gathering in the same region. Instead, we can use time thresholds, scoreboard and timestamp of the session to determine.

Following are specific explanations for the session:

1. Jungle: the hotspot must in the area of jungle, and the time of jungling must satisfy the time threshold range of the session of jungling. For example, when hitting the red BUFF, the residence time in the region must between ten seconds and fifteen seconds.

2. Being counter jungle: the hotspot must be in the area of jungling, and within the certain range of time, hotspots of both sides are in the same region. For example, when hitting the red BUFF, within the time that our team stay in the area of the red BUFF while the enemy exist over two points in the area of the red BUFF.

3. The failure of pulling: the hotspot must in the region of jungling and the range of jungling time will not within the time threshold of the session of jungling. At the same time the number of killing monsters is lower than the maximum number of existing monsters. For example, when hitting the red BUFF, the residence time in the region is higher than fifteen seconds or lower than ten seconds and the number of killing monsters showed on the scoreboard is lower than zero.

4. Being killed by monsters: when the player of jungling is going on the session of being killed by monsters, its hotspot must be in the region of jungling, and the data on the scoreboard must create death within the range of time threshold. For example, when hitting the red BUFF, the scoreboard generates death.

5. GANK: its hotspot must exist in the range of the mid-way and the residence time must satisfy the range of time threshold of GANK. For example, when begin to GANK in the mid-way, the player of jungling must show up in the range of mid-way and the residence time must between eight seconds and fourteen second.

6. Squatting for people: its hotspot must exist in the range of surrounding grass coordinates of every road and the residence time must between six seconds and fourteen seconds.

7. Backing to the base: If the jungle stayed in one place over six seconds and the next point showed up in the range of highland, it would be determined to be the session of backing to the base.

8. The generation of killing: That is to say the scoreboard generates kill.

9. The generation of assists: That is to say the scoreboard generates assists.

\subsection{The session partitioning algorithm of jungle}

The hot data sets of jungle is: the original hot data sets is $\boldsymbol{X}_{\text {host }}=\left\{\boldsymbol{x}_{1}, \boldsymbol{x}_{2}, \boldsymbol{x}_{3}, \cdots, \boldsymbol{x}_{n}, k e y\right\}$. Of all, the selection of threshold in the hot region key. If the source of hot data is from the blue team, the threshold is zero, or it will be one. After the mark, $\boldsymbol{B}_{\text {host }}=\left\{\boldsymbol{b}_{1}, \boldsymbol{b}_{2}, \boldsymbol{b}_{3}, \cdots, \boldsymbol{b}_{n}\right\}$ is the hotspot of the blue team. $\boldsymbol{R}_{\text {host }}=\left\{\boldsymbol{r}_{1}, \boldsymbol{r}_{2}, \boldsymbol{r}_{3}, \cdots, \boldsymbol{r}_{n}\right\}$ is the hotspot of the red team. The data sets of the scoreboard of jungle is: $\boldsymbol{S}_{\text {board }}=\left\{\boldsymbol{s} \boldsymbol{c}_{1}, \boldsymbol{s \boldsymbol { c } _ { 2 }}, \cdots, \boldsymbol{s} \boldsymbol{c}_{n}\right\}, \boldsymbol{S}_{\text {board }}$ and $\boldsymbol{S}_{\text {board }}$ : the vector sequence is one-to-one correspondence. The classified sets of the range of the map: $\boldsymbol{D}_{\text {area }}=\left\{\boldsymbol{d}_{1}, \boldsymbol{d}_{2}, \boldsymbol{d}_{3}, \cdots, \boldsymbol{d}_{17}, \boldsymbol{d}_{18}, \boldsymbol{d}_{19}\right\}$, of all, the range of jungling area of the blue team is $\boldsymbol{d}_{1}$ between $\boldsymbol{d}_{6}$ and that of the red team is between $\boldsymbol{d}_{7}$ and $\boldsymbol{d}_{12}$. Main route are from $\boldsymbol{d}_{13}$ to $\boldsymbol{d}_{15}$. The grass next to main route are form $\boldsymbol{d}_{16}$ to $\boldsymbol{d}_{18}$. Highland region: the blue team is : 
$\boldsymbol{d}_{19}$ the red team is: $\boldsymbol{d}_{20}$. The sets of time threshold of jungling: $\boldsymbol{T}_{\text {jungle }}=\left\{\boldsymbol{t}_{1}, \boldsymbol{t}_{2}, \boldsymbol{t}_{3}, \cdots, \boldsymbol{t}_{6}\right\}$, $\boldsymbol{t}_{1} \cdots \boldsymbol{t}_{6}$ sequence corresponding to $\boldsymbol{d}_{1} \cdots \boldsymbol{d}_{6}$, sequence corresponding to after adding six $\boldsymbol{d}_{7} \cdots \boldsymbol{d}_{12}$ The sets of time threshold of GANK of every lane: $\boldsymbol{M}_{\text {Gank }}=\left\{m_{1}, m_{2}, m_{3}\right\}$ The sets of time threshold of squatting for people of every lane: $\boldsymbol{Z}_{\text {Wait }}=\left\{\boldsymbol{z}_{1}, \boldsymbol{z}_{2} \boldsymbol{z}_{3}, \cdots, \boldsymbol{z}_{n}\right\}$ The classified sets of types of the session: $\boldsymbol{S}_{\text {session }}=\left\{s_{1}, s_{2}, s_{3}, \cdots, s_{9}\right\}$ The sets of results: $\boldsymbol{N}_{\text {result }}=\left\{\boldsymbol{n}_{l}, \boldsymbol{n}_{2}, \boldsymbol{n}_{3}, \cdots, \boldsymbol{n}_{m}\right\}, \boldsymbol{n}_{m}=\left\{\boldsymbol{x}_{1}, \boldsymbol{x}_{2}, \boldsymbol{x}_{3}, \cdots, \boldsymbol{x}_{n}, k_{m}, s_{m}\right\}$,

1. IF Key $=0$ : choose $\boldsymbol{d}_{1}$ to $\boldsymbol{d}_{6}$ mark $\boldsymbol{X}_{\text {host }}$ as $B_{\text {host }}$

2. ELSE : choose $\boldsymbol{d}_{7}$ to $\boldsymbol{d}_{12}$ and mark $\boldsymbol{X}_{\text {host }}$ as $R_{\text {host }}$

3. FOR every $\boldsymbol{d}_{i}$ in $\boldsymbol{D}_{\text {area }}$

4. FOR every point in $\boldsymbol{d}_{i}$

5. point is used as a cluster

6. FOR every cluster

7. IF exists difference $=1$ between 2 points in different clusters

8. combine 2 clusters

9. After combining, clusters are $\boldsymbol{N}_{\text {result }}=\left\{\boldsymbol{n}_{l}, \boldsymbol{n}_{2}, \boldsymbol{n}_{3}, \cdots, \boldsymbol{n}_{m}\right\}$, and $\boldsymbol{n}_{m}=\left\{\boldsymbol{x}_{1}, \boldsymbol{x}_{2}, \boldsymbol{x}_{3}, \cdots, \boldsymbol{x}_{i}, k_{i}, s_{i}, d_{i}\right\}, k_{i}$ is $\max \left(\right.$ sequense) - $\min \left(\right.$ sequense) in every $\boldsymbol{n}_{i}$

10. Must input blue and red point data of jungle

11. JUNGLE Area Analysis begin:

12. FOR every $\boldsymbol{n}_{m}$ in $\boldsymbol{N}_{\text {result }}$

13. IF $t_{i 0} \leq k_{m} \leq t_{i 1} s_{i}=$ 'fail to pull'

14. IF in $k_{m}$

15. IF $\boldsymbol{b}_{i}$ and $\boldsymbol{r}_{i}$ in the same $\boldsymbol{d}_{i} s_{i}=$ 'counter - jungle'

16. ELSE IF our jungle is dead THEN $s_{i}=$ 'executed'

17. IF $k_{m}>\max \left(\boldsymbol{t}_{n}\right)$

18. IF in the same $k_{m}$

19. IF $s c_{(i+1) l}-s c_{i l} \geq 1$ THEN $s_{i}=$ 'executed'

20. ELSE IF our jungle isn't dead THEN $s_{i}=$ 'counter - jungle'

21. IF percent of monsters that jungle killed in $k_{m}$ that $t_{i 0} \leq k_{m} \leq t_{i 1}$ do not reach $100 \%$ THEN $s_{i}=$ 'fail to pull'

22. ELSE THEN break;

23. IF $k_{m}>\max \left(\boldsymbol{t}_{n}\right)$

24. IF in the same $k_{m}$

25. IF $s c_{(i+1) l}-s c_{i l} \geq 1$ THEN $s_{i}=$ 'executed'

26. ELSE THEN $s_{i}=$ 'counter - jungle'

27. ELSE IF the next point of $\max \left(\right.$ sequence) point in $d_{i-1}$ or $d_{i+1}$ THEN break;

28. FOR every $\boldsymbol{n}_{m}$ in $\boldsymbol{d}_{13}$ to $\boldsymbol{d}_{15}$ (GANK Area Analysis begin)

29. IF $m_{i 0} \leq k_{m} \leq m_{i 1}$

30. THEN $s_{i}=$ 'gank'

31. FOR every $\boldsymbol{n}_{m}$ in $\boldsymbol{d}_{16}$ to $\boldsymbol{d}_{15}$ 
32. IF $z_{i 0} \leq k_{m} \leq z_{i 1}$ THEN $s_{i}=$ 'wait-gank'

33. FOR every point (Kill Analysis begin)

34. IF $s c_{(i+1) l}-s c_{i l} \geq 1$ THEN $s_{i}=$ 'slain-enemy'

35. Assist Analysis begin :

36. FOR every point

37. IF $s c_{(i+1) 3}-s c_{i 3} \geq 1$ THEN $s_{i}=$ 'assist-slain'

38. FOR every point(Back Analysis begin)

39. IF exists $x_{i}=x_{i+1}=x_{i+2}$ and next point of $x_{i+2}$ appear in $\boldsymbol{d}_{19}$ or $\boldsymbol{d}_{20}$ (judge by key) TNEN $s_{i}=$ 'back-city'

\subsection{Algorithm analysis}

In summary, the algorithm described in this article divides all actions of a role into the following 9 sessions:

1. Jungling: Hotspot activities in the range of $\boldsymbol{d}_{1}$ to $\boldsymbol{d}_{6}$ and $\boldsymbol{d}_{7}$ to $\boldsymbol{d}_{12}$, the activity time meets the time threshold $\boldsymbol{T}_{\text {jungle }}$, the percentage of monsters killed by jungle reaches $100 \%$. Jungling should be carried out in a certain field, and there is a certain time limit. Beyond or below this time range, it is clear that it is not pure jungling.

2. Counter-jungle: Hotspot activities in the range of $\boldsymbol{d}_{1}$ to $\boldsymbol{d}_{6}$ and $\boldsymbol{d}_{7}$ to $\boldsymbol{d}_{12}$, In the same time range, the enemy hotspot and our hotspot are in the same $\boldsymbol{d}_{n}$.

3. Fail to jungle: Hotspot activities in the range of $\boldsymbol{d}_{1}$ to $\boldsymbol{d}_{6}$ and $\boldsymbol{d}_{7}$ to $\boldsymbol{d}_{12}$, In the same time range, the enemy hotspot and our hotspot are not in the same $\boldsymbol{d}_{n}$, the percentage of monsters killed by jungle does not reach $100 \%$.

4. Killed by the monster(executed) : Hotspot activities in the range of $\boldsymbol{d}_{1}$ to $\boldsymbol{d}_{6}$ and $\boldsymbol{d}_{7}$ to $\boldsymbol{d}_{12}$, In the same time range, the enemy hotspot and our hotspot are not in the same $\boldsymbol{d}_{n}$ and our jungle dies .

5. Slay the enemy: The scoreboard data shows that someone kill the enemy.

6. Assists: The scoreboard data shows that someone help teammate to kill the enemy.

7. GANK: The stay time on the main route is in line with $m_{n}$.

8. Wait to GANK: The stay time in the grass next to the main route is in line with $\boldsymbol{z}_{n}$.

9. Back: Hotspots stay somewhere over two sequence values, and the next point of the maximum sequence appear birth place.

The algorithm involves three main parameters: time threshold, range threshold and socreboard. In order to narrow down the range of judgment and to determine exactly where the behavior is occurring, we introduce the range threshold. At the same time, in order to segment adjacent sessions, we introduce the time threshold as the segmentation point. Scoreboard helps algorithm deal with simillar session.,for example:'fail to jungle' and 'executed'. If they were all happen beyond the time threshold, It is impossible to distinguish the two sessions without the introduction of the scoreboard because the information displayed in the coordinates is the same with the two behaviors. At last, only scoreborad can be given accurately but the time threshold and range threshold must be given according to different E-sports Game.

Algorithm 1-14 is the data initialization phase of the algorithm, and all points are classified according to the range threshold. 
Algorithm 15-39, jungle stay in the field of jungling to do 4 behaviors : jungling, counter-jungle, fail to jungle, executed. Session happened in the same field can not be distinguish by points, so we use time threshold to solve this problem. Then we can get two sessions: executed and counter-jungle. At last, we introduce scoreboard to distinguish 'fail to jungle'.In summary, we succeed in distinguishing 4 sessions happened in the field of jungling.

Algorithm 40-57, we We differentiate the remaining five sessions. In GANK analysis, jungle must GANK or find chance to GANK when the time that jungle stay in main routes meets time threshold.Sp we propose to use two time threshold to dertermine whether it is GANK or 'Wait to GANK'.We just decide that session is 'slay the enemy' or 'assists' and do not need to make a regional decision because Back can be judge by scorebaard directly and happen everywhere in the map. When judge 'Back', the person that uses the algorithm need to set time threshold according to different game. It is like 'slay the enemy' and 'assists' that happens every in the map, so we do not need to use range threshold.

Based on the above analysis, the algorithm proposed in this paper solves the problem of session identification in E-sports.

\subsection{The results and analysis of experimental data.}

To verify the recognition effect of the session of the e-sports, the paper gives data sets of a series of five competitions. To data sets of every battlefield, we are going on the exporting test for the recognition of the session to verify the validity of the algorithm. The source of data is from the recording of videos of LOL S8 season. From the data preprocessing we conclude that: the number of competition data, the sets of coordinates of both sides (jungle) (the sequence of coordinates represents timestamp: the interval among every coordinate point is three seconds, starts the game clock from 00:00), the sets of scoreboards of both sides (jungle).

Table 1. Session recognition results

\begin{tabular}{|c|c|c|c|c|c|}
\hline No. & $\begin{array}{c}\text { Blue Hot } \\
\text { data Set }\end{array}$ & $\begin{array}{c}\text { Red Hot data } \\
\text { Set }\end{array}$ & $\begin{array}{c}\text { Blue } \\
\text { Scoreboard Set }\end{array}$ & $\begin{array}{c}\text { Red } \\
\text { Scoreboard Set }\end{array}$ & $\begin{array}{c}\text { The correct rate of } \\
\text { division }\end{array}$ \\
\hline 1 & BHotSet1 & RHotSet1 & BscSet1 & RscSet1 & $94 \%$ \\
\hline 2 & BHotSet2 & RhotSet2 & BscSet2 & RscSet2 & $93 \%$ \\
\hline 3 & BHotSet3 & RhotSet3 & BscSet3 & RscSet3 & $91 \%$ \\
\hline 4 & BhotSet4 & RhotSet4 & RhotSet4 & RscSet4 & $92 \%$ \\
\hline 5 & BHotSet5 & RhotSet5 & BscSet5 & RscSet5 & $90 \%$ \\
\hline
\end{tabular}

As is shown in table 1, it shows the experimental data of the series of five competitions and the accuracy of the algorithm of the session that put forward in this paper. Of all, explanations in turn from the header are: the number of the data of the competition, the sets of hot data of the blue team, the sets of hot data of the red team, the data sets of the scoreboard of the blue team, the data sets of the scoreboard of the red team, the accuracy of the recognition of the session. Each line of the sheet is respectively the name of the each data sets and results of accuracy that represented by percentages.

By the analysis of results of the experimental data, this algorithm obtain the more precise accuracy of recognition.

\section{Conclusion}

The paper put forward a session partitioning algorithm of the character (jungle) in the esports. It has solved the important problem that the analysis of behavior patterns of the esports' individual character not solved directly and solved by anyone. And has put forward 
the session partitioning algorithm based on the coordinate, timestamp, scoreboard data exported through videos of jungling. It adopts the clustering prototype thinking that partitions spatial range in advance and then determines by the range of time threshold to solve the problem of the character session which is difficult to be partitioned. The experimental results indicate that the algorithm in the paper has good effects on the recognition of the session of the character in the e-sports. However, to some special players or heroes, the determination has a few errors. At the same time, whether we can figure out the time threshold and range threshold rather than give artificially and how to make these conjoint analysis of the session export accurate tactical instruction [10] by this algorithm is a problem, which we still keep on studying.

\section{Acknowledgment}

This article is funded by the Jinling Institute of Science and Technology, a high-level talent research startup fund, and a web user behavior analysis and research project based on quantum algorithms(No. jit-b-201624) and the Social Engineering Network Behavior Analysis(No.2017YFB0802800).

\section{References}

1. François Rioult, Jean-Philippe Métivier, Boris Helleu, Nicolas Scelles, Christophe Durand. Mining Tracks of Competitive Video Games[J]. AASRI Procedia,2014, 8.

2. Yili Zhu. Research on Association Rules Algorithm and Its Application in Electronic Sports. [D]. Application in Electronic Sports, 2017.

3. Huijing Hao, Xianwen Fang, Lili, Xiangwei Liu. Analysis of Effective Low-frequency Behavioural Patterns Based on Petri Net Behaviour Closeness [J/OL]. Computer Science, 2019(02):1-5[2018-0422].http://kns.cnki.net/kcms/detail/50.1075.TP.20180416.0922.002.html.

4. E. Iorgulescu,V.A. Voicu,C. Sârbu,F. Tache,F. Albu,A. Medvedovici. Experimental variability and data pre-processing as factors affecting the discrimination power of some chemometric approaches (PCA, CA and a new algorithm based on linear regression) applied to (+/-)ESI/MS and RPLC/UV data: Application on green tea extracts[J]. Talanta,2016,155.

5. Hongju Cheng,Danyang Feng,Xiaobin Shi,Chongcheng Chen. Data quality analysis and cleaning strategy for wireless sensor networks[J]. EURASIP Journal on Wireless Communications and Networking,2018,2018(1).

6. Murat Semerci,Ali Taylan Cemgil,Bülent Sankur. An intelligent cyber security system against DDoS attacks in SIP networks[J]. Computer Networks,2018,136.

7. Bahjat Fatima,Huma Ramzan,Sohail Asghar. Session identification techniques used in web usage mining[J]. Online Information Review,2016,40(7).

8. Chen Wei, Wei Zi. Research on Market Positioning of Video Game Products - A Comparative Analysis Based on DOTA and LOL[J]. Computer Knowledge and Technology, 2016,12(32):281-284.

9. Yibin Ren. China e-sports LOL China S5 format - factor analysis in the quarter - S5 season season in the final in 2015, for example [D]. Northwest Normal University, 2016.

10. Luis Fernando Cruz Quiroga, Wilfrido Alejandro Moreno. Neural Networks and Computational Complexity [M].Elsevier Inc.:2016-06-15. 